\title{
The dissipation approximation and viscous potential flow
}

\author{
By D. D. JOSEPH AND J. WANG \\ Department of Aerospace Engineering and Mechanics, University of Minnesota, \\ Minneapolis, MN 55455, USA
}

(Received 18 June 2003 and in revised form 1 February 2004)

Dissipation approximations have been used to calculate the drag on bubbles and drops and the decay rate of free gravity waves on water. In these approximations, viscous effects are calculated by evaluating the viscous stresses on irrotational flows. The pressure is not involved in the dissipation integral, but it enters into the power of traction integral, which equals the dissipation. A viscous correction of the irrotational pressure is needed to resolve the discrepancy between the zero-shear-stress boundary condition at a free surface and the non-zero irrotational shear stress. Here we show that the power of the pressure correction is equal to the power of the irrotational shear stress. The viscous pressure correction on the interface can be expressed by a harmonic series. The principal mode of this series is matched to the velocity potential and its coefficient is explicitly determined. The other modes do not enter into the expression for the drag on bubbles and drops. They vanish in the case of free gravity waves.

\section{Introduction}

The drag on a spherical gas bubble of radius $a$ rising in a viscous liquid at high Reynolds number has been calculated by various investigators beginning with Levich (1949), who obtained the value $12 \pi a \mu U$ or equivallently the drag coefficient $48 / R$, where $R=2 a U \rho / \mu$ is the Reynolds number, by calculating the dissipation of the irrotational flow around the bubble. Moore (1959) calculated the drag directly by integrating the pressure and viscous normal stress of the potential flow and neglecting the viscous shear stress (which physically should be zero), obtaining the value $8 \pi a \mu U$. The discrepancy between these two values led G. K. Batchelor, as reported in Moore (1963), to suggest the idea of a pressure correction to the irrotational pressure. In that paper, Moore performed a boundary layer analysis and his pressure correction is readily obtained by setting $y=0$ in his equation (2.37):

$$
p_{\mathrm{v}}=(4 / R)(1-\cos \theta)^{2}(2+\cos \theta) / \sin ^{2} \theta,
$$

which is singular at the separation point where $\theta=\pi$. The presence of separation is a problem for the application of boundary layers to the calculation of drag on solid bodies. To find the drag coefficient Moore calculated the momentum defect, and obtained the Levich value $48 / R$ plus contributions of order $R^{-3 / 2}$ or lower. None of these drag formulae are in convincing agreement with experiments (see Batchelor 1967, figure 5.14.1 and Joseph 2003, figure 3), because the deviation of the bubble from sphericity occurs at much lower Reynolds number than would be required for the validity of any boundary layer theory. Obviously it would be of interest to 
calculate the drag directly by solving the boundary layer equations and hence obtain the drag from the corrected pressure. However, this has so far not been accomplished.

Kang \& Leal (1988) put the shear stress of the potential flow on the bubble surface to zero and calculate a pressure correction. They obtain the drag coefficient given by Levich's dissipation approximation by direct integration of the normal viscous stress and pressure over the bubble surface. They accomplished this by expanding the pressure correction as a spherical harmonic series and noting that only one term of this series contributes to the drag, no appeal to the boundary-layer approximation being necessary.

A different application of the dissipation approximation was presented by Lamb (1932). He computed the effect of viscosity on the decay of small-amplitude progressive free waves on water. This problem falls into the general framework presented here; we compute a viscous correction to the irrotational pressure which can be used to correct the direct calculation of decay using viscous potential flow. Fortunately, Lamb also obtained an exact solution from which the approximations can be evaluated and the nature of the boundary layer can be rigorously examined.

\section{Pressure correction formulae}

The mechanical energy equation for the Navier-Stokes equations is

$$
\frac{\mathrm{d}}{\mathrm{d} t} \int_{V} \frac{\rho u^{2}}{2} \mathrm{~d} V=\int_{A} \boldsymbol{u} \cdot(\boldsymbol{T} \cdot \boldsymbol{n}) \mathrm{d} A-\int_{V} 2 \mu \boldsymbol{D}: \boldsymbol{D} \mathrm{d} V
$$

where

$$
\boldsymbol{T}=-p \mathbf{1}+2 \mu \boldsymbol{D}[\boldsymbol{u}]
$$

is the stress, $\boldsymbol{D}[\boldsymbol{u}]$ is the rate of strain tensor, $V$ is the volume occupied by the fluid, $A$ is the boundary of $V$, and $\boldsymbol{n}$ is the outward normal of $V$ on $A$. When the kinetic energy flux across the boundary is zero and the flow is steady, (2.1) becomes

$$
\mathscr{P} \equiv \int_{A} \boldsymbol{u} \cdot(\boldsymbol{T} \cdot \boldsymbol{n}) \mathrm{d} A=\int_{V} 2 \mu \boldsymbol{D}: \boldsymbol{D} \mathrm{d} V \equiv \mathscr{D}
$$

where $\mathscr{D}$ is the dissipation and $\mathscr{P}$ is the power of traction.

We consider irrotational solutions of the Navier-Stokes equations with $\boldsymbol{u}=\nabla \phi$ and stresses given by (2.2) where $p=p_{\mathrm{i}}$ is the pressure function which is generated by the irrotational flow of an inviscid fluid. We call this solution viscous potential flow $(\mathrm{VPF})$ :

$$
\boldsymbol{u}=\nabla \phi, \quad \nabla^{2} \phi=0, \quad \boldsymbol{T}=-p_{\mathrm{i}} \mathbf{1}+2 \mu \nabla \otimes \nabla \phi
$$

in the bulk liquid. The contribution of $p_{i}$ to the drag on bodies in steady irrotational flow of an inviscid fluid vanishes (D'Alembert paradox). In general, the normal stress

$$
\tau_{\mathrm{n}}=\boldsymbol{n} \cdot 2 \mu \nabla \otimes \nabla \phi \cdot \boldsymbol{n} \neq 0
$$

and the shear stress

$$
\tau_{\mathrm{s}}=\boldsymbol{t} \cdot 2 \mu \nabla \otimes \nabla \phi \cdot \boldsymbol{n} \neq 0
$$

do not vanish at the gas-liquid interface. Here $t$ is the unit tangential vector.

We next consider a viscous correction of the above solution. The problem solved by this correction expresses the idea, frequently encountered in the literature, that a viscous correction of the pressure at the gas-liquid interface is required to compensate for the irrotational shear stress; instead of $\tau_{\mathrm{s}} \neq 0$, we have $\tau_{\mathrm{s}}=0$. We call this problem 
the viscous correction of VPF (VCVPF):

$$
\boldsymbol{u}=\nabla \phi, \quad \nabla^{2} \phi=0, \quad \boldsymbol{T}=-p \mathbf{1}+2 \mu \nabla \otimes \nabla \phi
$$

in the bulk liquid; at the gas-liquid interface

$$
\begin{aligned}
& \tau_{\mathrm{n}}=\boldsymbol{n} \cdot 2 \mu \nabla \otimes \nabla \phi \cdot \boldsymbol{n} \neq 0, \\
& \tau_{\mathrm{s}}=0, \\
& p=p_{\mathrm{i}}+p_{\mathrm{v}},
\end{aligned}
$$

where $p_{\mathrm{v}}$ is a viscous pressure correction to the irrotational pressure $p_{\mathrm{i}}$.

Of course, the viscous dissipation computed for VPF and VCVPF are identical because only the strain rate in the bulk liquid enters into the dissipation integral and the strain rate is computed on the same velocity potential in the two problems.

We compute the power of the traction integral of the VPF which is equal to the dissipation according to (2.3):

$$
\mathscr{D}=\mathscr{P}=\int_{A} \boldsymbol{u} \cdot(\boldsymbol{T} \cdot \boldsymbol{n}) \mathrm{d} A=\int_{A}\left[\boldsymbol{u} \cdot \boldsymbol{n}\left(-p_{\mathrm{i}}+\tau_{\mathrm{n}}\right)+\boldsymbol{u} \cdot \boldsymbol{t} \tau_{\mathrm{s}}\right] \mathrm{d} A .
$$

The power of the traction integral of VCVPF can be computed using (2.8)-(2.10); we assume that the integral is equal to the dissipation (up to the leading order $1 / R$ ):

$$
\mathscr{D}=\mathscr{P}=\int_{A} \boldsymbol{u} \cdot(\boldsymbol{T} \cdot \boldsymbol{n}) \mathrm{d} A=\int_{A} \boldsymbol{u} \cdot \boldsymbol{n}\left(-p_{\mathrm{i}}-p_{\mathrm{v}}+\tau_{\mathrm{n}}\right) \mathrm{d} A .
$$

Since $\mathscr{D}, \boldsymbol{u}, p_{\mathrm{i}}$ and $\tau_{\mathrm{n}}$ are the same in (2.11) and (2.12), we find that

$$
\int_{A} \boldsymbol{u} \cdot \boldsymbol{n}\left(-p_{\mathrm{v}}\right) \mathrm{d} A=\int_{A} \boldsymbol{u} \cdot \boldsymbol{t} \tau_{\mathrm{s}} \mathrm{d} A=\mathscr{P}_{\mathrm{s}},
$$

where $\mathscr{P}_{\mathrm{s}}$ denotes the power of the irrotational shear stress. Equation (2.13) is the main result relating the irrotational shear stress to the pressure correction.

We consider separable solutions of $\nabla^{2} \phi=0$. For simplicity, we consider axisymmetric or planar problems and use the orthogonal coordinate system $(\alpha, \beta)$; the gasliquid interface is given by $\alpha=$ const. The solution of the potential flow equations may be written as

$$
\phi=h_{k}(\alpha) f_{k}(\beta),
$$

where $f_{k}(\beta)$ is the $k$ th mode of the surface harmonics. A pressure correction function which is periodic or finite at the gas-liquid interface may be expanded as a series of surface harmonics of integral orders:

$$
-p_{\mathrm{v}}=C_{k} f_{k}(\beta)+\sum_{j \neq k} C_{j} f_{j}(\beta),
$$

where $f_{j}(\beta)$ are surface harmonics and the $C_{j}$ are constant coefficients.

Substitution of (2.15) into (2.13) leads to

$$
C_{k} \int_{A} \boldsymbol{u} \cdot \boldsymbol{n} f_{k}(\beta) \mathrm{d} A+\sum_{j \neq k} C_{j} \int_{A} \boldsymbol{u} \cdot \boldsymbol{n} f_{j}(\beta) \mathrm{d} A=\mathscr{P}_{\mathrm{s}} .
$$

We assume that the normal velocity is orthogonal to $f_{j}(\beta), j \neq k$ :

$$
\int_{A} \boldsymbol{u} \cdot \boldsymbol{n} f_{j}(\beta) \mathrm{d} A=0 \quad \text { when } \quad j \neq k
$$


which is a verifiable condition and is confirmed in each example we consider here. Now (2.16) gives the coefficient $C_{k}$

$$
C_{k}=\frac{\mathscr{P}_{\mathrm{s}}}{\int_{A} \boldsymbol{u} \cdot \boldsymbol{n} f_{k}(\beta) \mathrm{d} A} .
$$

Using (2.18) for VCVPF we may write

$$
-p=-p_{\mathrm{i}}+\frac{\mathscr{P}_{\mathrm{s}} f_{k}(\beta)}{\int_{A} \boldsymbol{u} \cdot \boldsymbol{n} f_{k}(\beta) \mathrm{d} A}+\sum_{j \neq k} C_{j} f_{j}(\beta) .
$$

The term $\mathscr{P}_{\mathrm{s}} f_{k}(\beta) / \int_{A} \boldsymbol{u} \cdot \boldsymbol{n} f_{k}(\beta) \mathrm{d} A$ may be called the principal part of the viscous pressure correction; it is proportional to the power integral of the uncompensated irrotational shear stress. It is the only term in the pressure correction to enter into the power of traction integral, into the direct calculation of the drag on rising bubbles or drops, and into the expression for the decay rate of free gravity waves. The principal part of the pressure correction is explicitly computable as we shall see in the examples to follow. For the free wave problem we shall show that $C_{j}=0$ when $j \neq k$ (see $\S 6.4$ ). In general, the values of $C_{j}, j \neq k$ are not known, but for the special case of a rising spherical gas bubble, Kang \& Leal (1988) presented computable expressions for these coefficients.

The expression (2.19) completes the formulation of equations (2.7)-(2.10) for VCVPF up to the principal part of the harmonic series.

We may compare the dissipation calculation and direct calculation of the drag using VPF defined by (2.4)-(2.6) and VCVPF defined by (2.7)-(2.10) and (2.19). Let $D_{1}$ be the drag calculated by the dissipation method

$$
D_{1}=\mathscr{D} / U
$$

and $D_{2}$ be the drag from direct calculation

$$
D_{2}=\int_{A} \boldsymbol{e}_{x} \cdot \boldsymbol{T} \cdot \boldsymbol{n} \mathrm{d} A=\int_{A}\left[\boldsymbol{e}_{x} \cdot \boldsymbol{n}\left(-p+\tau_{\mathrm{n}}\right)+\boldsymbol{e}_{x} \cdot \boldsymbol{t} \tau_{\mathrm{s}}\right] \mathrm{d} A,
$$

where $x$ is the direction of translation. The direct calculation using VPF leads to $D_{2}=0$ even though the dissipation is not zero, which is a known result (see, for example, Zierep 1984; Joseph \& Liao 1994). The dissipation approach involves a volume integral and the direct calculation involves a surface integral. The solution to the Navier-Stokes equations in these nearly irrotational flows involves a leading-order term (the irrotational solution) and a viscous correction at the boundary. When using the dissipation approach, the leading-order calculation only involves the irrotational result. However, the viscous correction has to be considered to obtain the leadingorder result when using the direct calculation. We shall focus on the direct calculation using VCVPF in the following examples $(\S \S 3-5)$ and show that $D_{2}$ computed using VCVPF here is equal to $D_{1}$ obtained by the dissipation method in the literature.

\section{Rising spherical gas bubble}

Consider now a spherical gas bubble rising with a constant velocity $U \boldsymbol{e}_{x}$ in a viscous fluid, for which

$$
\phi=-\frac{1}{2} U a^{3} \frac{\cos \theta}{r^{2}}
$$


At the surface of the bubble, where $r=a$, we have

$$
\left.\begin{array}{l}
u_{r}=U \cos \theta, \quad u_{\theta}=U \sin \theta / 2 ; \\
\tau_{r r}=-6 \mu U \cos \theta / a, \quad \tau_{r \theta}=-3 \mu U \sin \theta / a ; \\
p_{\mathrm{i}}=p_{\infty}+\frac{1}{2} \rho U^{2}\left(1-\frac{9}{4} \sin ^{2} \theta\right) .
\end{array}\right\}
$$

The dissipation is given by $\mathscr{D}=12 \pi \mu a U^{2}$ and $\mathscr{P}_{\mathrm{s}}=4 \pi \mu a U^{2}$.

The pressure correction may be expanded as a spherical surface harmonic series $\sum_{j=0}^{\infty} C_{j} P_{j}(\cos \theta)$. Substitution of $u_{r}$ and $p_{\mathrm{v}}$ into (2.13) gives

$$
-\int_{-1}^{1} U P_{1}(\cos \theta)\left[C_{1} P_{1}(\cos \theta)+\sum_{j \neq 1} C_{j} P_{j}(\cos \theta)\right] 2 \pi a^{2} \mathrm{~d}(\cos \theta)=\mathscr{P}_{\mathrm{s}} .
$$

The coefficient $C_{1}$ is then determined and the pressure correction is

$$
-p_{\mathrm{v}}=-3 \mu U P_{1}(\cos \theta) / a+\sum_{j \neq 1} C_{j} P_{j}(\cos \theta),
$$

which is the same as the pressure correction of Kang \& Leal (1988) who obtained it by means of a general relationship between the viscous pressure correction and the vorticity distribution for a spherical bubble in an arbitrary axisymmetric flow. Kang \& Leal demonstrated that the drag from direct calculation using the pressure correction (3.4) is $12 \pi \mu a U$, the same as the drag by dissipation calculation.

\section{Rising oblate ellipsoidal bubble (Moore 1965)}

The equation of the ellipsoid is

$$
\frac{x^{2}+y^{2}}{b^{2}}+\frac{z^{2}}{a^{2}}=1
$$

where $b \geqslant a$. Orthogonal ellipsoidal coordinates $(\alpha, \beta, \omega)$ are related to $(x, y, z)$ by

$$
\begin{aligned}
& x=\kappa\left[\left(1+\alpha^{2}\right)\left(1-\beta^{2}\right)\right]^{1 / 2} \cos \omega, \\
& y=\kappa\left[\left(1+\alpha^{2}\right)\left(1-\beta^{2}\right)\right]^{1 / 2} \sin \omega, \\
& z=\kappa \alpha \beta .
\end{aligned}
$$

The ellipsoid is given by $\alpha=\alpha_{0}$ provided that

$$
\kappa\left(1+\alpha_{0}^{2}\right)^{1 / 2}=b, \quad \kappa \alpha_{0}=a .
$$

The potential for an oblate ellipsoid rising with a constant velocity $U \boldsymbol{e}_{z}$ is

$$
\phi=-U \kappa q \beta\left(1-\alpha \cot ^{-1} \alpha\right),
$$

where $q\left(\alpha_{0}\right)=\left(\cot ^{-1} \alpha_{0}-\alpha_{0} /\left(1+\alpha_{0}^{2}\right)\right)^{-1}$. The velocity components in the ellipsoidal coordinates are $\left(u_{\alpha}, u_{\beta}, 0\right)$, and at the surface of the ellipsoid, we have

$$
u_{\alpha}=U \beta \sqrt{\frac{1+\alpha_{0}^{2}}{\alpha_{0}^{2}+\beta^{2}}}, \quad u_{\beta}=-U q \sqrt{\frac{1-\beta^{2}}{\alpha_{0}^{2}+\beta^{2}}}\left(1-\alpha_{0} \cot ^{-1} \alpha_{0}\right) .
$$

The normal stress $\tau_{\alpha \alpha}$ and shear stress $\tau_{\beta \alpha}$ are calculated using the potential flow, and their values at the surface of the ellipsoid are

$$
\tau_{\alpha \alpha}=-2 \mu \frac{U \beta q\left(1+2 \alpha_{0}^{2}+\beta^{2}\right)}{\left(\alpha_{0}^{2}+\beta^{2}\right)^{2} \kappa\left(1+\alpha_{0}^{2}\right)}, \quad \tau_{\beta \alpha}=2 \mu \frac{U q \alpha_{0}}{\kappa\left(\alpha_{0}^{2}+\beta^{2}\right)^{2}} \sqrt{\frac{1-\beta^{2}}{1+\alpha_{0}^{2}}} .
$$


Then the power of the shear stress can be evaluated:

$$
\mathscr{P}_{\mathrm{s}}=-\int_{A} u_{\beta} \tau_{\beta \alpha} \mathrm{d} A=4 \mu \pi U^{2} \kappa q^{2}\left(1-\alpha_{0} \cot ^{-1} \alpha_{0}\right)\left[\alpha_{0}+\left(1-\alpha_{0}^{2}\right) \cot ^{-1} \alpha_{0}\right] / \alpha_{0}^{2} .
$$

Now we calculate the pressure correction $p_{\mathrm{v}}$. Noting that ellipsoidal harmonics $P_{j}(\beta)$ (see Lamb 1932) are appropriate in this case and the potential (4.1) is proportional to $P_{1}(\beta)=\beta$, we write the pressure correction as

$$
-p_{\mathrm{v}}=C_{1} P_{1}(\beta)+\sum_{j \neq 1} C_{j} P_{j}(\beta) .
$$

Inserting (4.5) into (2.13) and using $\mathrm{d} A=2 \pi \kappa^{2}\left(1+\alpha_{0}^{2}\right)^{1 / 2}\left(\alpha_{0}^{2}+\beta^{2}\right)^{1 / 2} \mathrm{~d} \beta$, we obtain

$$
-\int_{A} u_{\alpha}\left(-p_{\mathrm{v}}\right) \mathrm{d} A=-2 \pi \kappa^{2} U\left(1+\alpha_{0}^{2}\right) \int_{-1}^{1} P_{1}(\beta)\left[C_{1} P_{1}(\beta)+\sum_{j \neq 1} C_{j} P_{j}(\beta)\right] \mathrm{d} \beta=\mathscr{P}_{\mathrm{s}} .
$$

The terms $P_{j}(j \neq 1)$ do not contribute the integral; the coefficient $C_{1}$ is determined. Then the pressure correction is

$$
-p_{\mathrm{v}}=\frac{-3 \mu U q^{2}}{\kappa\left(1+\alpha_{0}^{2}\right) \alpha_{0}^{2}}\left(1-\alpha_{0} \cot ^{-1} \alpha_{0}\right)\left[\alpha_{0}+\left(1-\alpha_{0}^{2}\right) \cot ^{-1} \alpha_{0}\right] P_{1}(\beta)+\sum_{j \neq 1} C_{j} P_{j}(\beta) .
$$

At the limit $\alpha_{0} \rightarrow \infty$ where the ellipsoid becomes a sphere, the pressure correction (4.7) reduces to

$$
\lim _{\alpha_{0} \rightarrow \infty}-p_{\mathrm{v}}=-3 \mu U \cos \theta / a+\sum_{j \neq 1} C_{j} P_{j}(\cos \theta)
$$

with $\beta=\cos \theta$ at this limit being understood. This is in agreement with the pressure correction (3.4) for the spherical gas bubble.

We calculate the drag by direct integration:

$$
D_{2}=\int_{A} \boldsymbol{e}_{z} \cdot \boldsymbol{e}_{\alpha}\left(-p_{\mathrm{v}}+\tau_{\alpha \alpha}\right) \mathrm{d} A=\frac{4 \mu \pi U \kappa q^{2}}{1+\alpha_{0}^{2}}\left(\frac{1}{\alpha_{0}}+\frac{1-\alpha_{0}^{2}}{\alpha_{0}^{2}} \cot ^{-1} \alpha_{0}\right),
$$

which is in agreement with the dissipation calculation of Moore (1965).

\section{A liquid drop rising in another liquid (Harper \& Moore 1968)}

The steady flow of a spherical liquid drop in another immiscible liquid can be approximated by Hill's spherical vortex inside, and potential flow outside. We use the superscript $o$ for quantities outside the drop and $i$ for quantities inside. The stream and potential functions of the outer flow are

$$
\psi^{o}=\frac{1}{2} U \sin ^{2} \theta \frac{a^{3}}{r} \quad \text { and } \quad \phi=-\frac{1}{2} U a^{3} \frac{\cos \theta}{r^{2}},
$$

respectively. The stream function for a Hill's vortex moving at a constant velocity relative to fixed coordinate system is

$$
\psi^{i}=\frac{3 U r^{2}}{4} \sin ^{2} \theta\left(1-\frac{r^{2}}{a^{2}}\right)+\frac{1}{2} U r^{2} \sin ^{2} \theta=\frac{U r^{2}}{4} \sin ^{2} \theta\left(5-\frac{3 r^{2}}{a^{2}}\right) .
$$


At the surface of the drop, where $r=a$, we have

$$
\begin{gathered}
u_{r}=u_{r}^{o}=u_{r}^{i}=U \cos \theta, \quad u_{\theta}=u_{\theta}^{o}=u_{\theta}^{i}=U \sin \theta / 2, \\
\tau_{r r}^{o}=-6 \mu^{o} U \cos \theta / a, \quad \tau_{r \theta}^{o}=-3 \mu^{o} U \sin \theta / a, \\
\tau_{r r}^{i}=-6 \mu^{i} U \cos \theta / a, \quad \tau_{r \theta}^{i}=9 \mu^{i} U \sin \theta /(2 a) .
\end{gathered}
$$

This Hill's vortex problem fits in with the general framework discussed in $\S 2$ in the sense that there is a shear stress discontinuity at the interface which needs to be resolved by adding a pressure correction to the irrotational pressure. However, it is somewhat different from gas-liquid interface problems because the shear stress inside the drop is not zero but is determined by the Hill's vortex. Again we seek the expression for the pressure correction by comparing the VPF solution and the VCVPF solution. We proceed by calculating the total dissipation of the system, which is equal to the sum of the power of traction on the outer and inner liquids $\mathscr{P}^{o}+\mathscr{P}^{i}$. There is only one way to calculate $\mathscr{P}^{i}$, but $\mathscr{P}^{o}$ may be evaluated on VPF or VCVPF. For VPF, $\tau_{r r}^{o}$ and $\tau_{r \theta}^{o}$ given by (5.4) are used to calculate $\mathscr{P}^{o}$ and

$$
\mathscr{D}=\mathscr{P}^{o}+\mathscr{P}^{i}=-\int_{A}\left[u_{r}\left(-p_{\mathrm{i}}+\tau_{r r}^{o}\right)+u_{\theta} \tau_{r \theta}^{o}\right] \mathrm{d} A+\mathscr{P}^{i} .
$$

For VCVPF, a pressure correction is added to resolve the discontinuity between $\tau_{r \theta}^{o}$ and $\tau_{r \theta}^{i}$. Then the value of the shear stress at the interface is $\tau_{r \theta}^{i}$, not $\tau_{r \theta}^{o}$. The dissipation for VCVPF is

$$
\mathscr{D}=\mathscr{P}^{o}+\mathscr{P}^{i}=-\int_{A}\left[u_{r}\left(-p_{\mathrm{i}}-p_{\mathrm{v}}+\tau_{r r}^{o}\right)+u_{\theta} \tau_{r \theta}^{i}\right] \mathrm{d} A+\mathscr{P}^{i} .
$$

Since $\mathscr{D}, \mathscr{P}^{i}, p_{\mathrm{i}}, \tau_{r r}^{o}$ and $\boldsymbol{u}$ are the same in both cases, we find that

$$
\int_{A} u_{r}\left(-p_{\mathrm{v}}\right) \mathrm{d} A=\int_{A} u_{\theta}\left(\tau_{r \theta}^{o}-\tau_{r \theta}^{i}\right) \mathrm{d} A .
$$

Now we expand the pressure correction as a spherical surface harmonic series and (5.8) becomes

$$
\int_{-1}^{1} U \cos \theta\left[C_{1} \cos \theta+\sum_{j \neq 1} C_{j} P_{j}(\cos \theta)\right] 2 \pi a^{2} \mathrm{~d}(\cos \theta)=-4 \pi a U^{2}\left(\mu^{o}+3 \mu^{i} / 2\right) .
$$

The coefficient $C_{1}$ is then obtained and the pressure correction is

$$
-p_{\mathrm{v}}=\frac{-3 U}{a}\left(\mu^{o}+\frac{3 \mu^{i}}{2}\right) \cos \theta+\sum_{j \neq 1} C_{j} P_{j}(\cos \theta) .
$$

If the inside liquid is gas, $\mu^{i}=0$ and the first term of (5.10) becomes $-3 \mu^{o} U \cos \theta / a$, which agrees with the first term of the pressure correction (3.4) for a gas bubble. The pressure correction (5.10) can also be tested by direct calculation of the $\operatorname{drag} \mathrm{D}_{2}$ on the drop:

$$
D_{2}=\int_{A} \boldsymbol{e}_{x} \cdot \boldsymbol{T} \cdot\left(-\boldsymbol{e}_{\mathrm{r}}\right) \mathrm{d} A=-\int_{A}\left[\cos \theta\left(-p_{\mathrm{v}}+\tau_{r r}^{o}\right)-\sin \theta \tau_{r \theta}^{i}\right] \mathrm{d} A=12 \pi a U\left(\mu^{o}+\frac{3 \mu^{i}}{2}\right)
$$

which is the same as the result from the dissipation approximation by Harper \& Moore (1968). 


\section{Decay of free gravity waves on water}

It is possible to have a progressive gravity wave of permanent form if the viscosity of the liquid below air is identically zero. Lamb $(1932, \S \S 348,349)$ performed an analysis of the effect of viscosity on these waves. The wave decays and the decay rate may be obtained in two ways: by a dissipation calculation or by a direct (stability) calculation using viscous potential flow. This is analogous to the dissipation and direct calculation of drag. The two decay rates are not the same.

Lamb also constructed an exact solution to this problem: it gives a decay rate different from the two just mentioned; it reduces to the one computed by the dissipation method for long waves $\left(k \ll\left(g / v^{2}\right)^{1 / 3}\right.$, where $k$ is the wavenumber) and to the one computed directly for short waves $\left(k \gg\left(g / v^{2}\right)^{1 / 3}\right)$. The dissipation method gives the wrong result for short waves and the direct method using VPF gives the wrong result for long waves.

Lamb's exact solution also reveals the vorticity near the wave surface, which provides explanations for the aforementioned discrepancies. At the long wave limit, the vorticity is important in a thin boundary layer; thus a pressure correction is needed. We calculate a pressure correction by the method given in $\S 2$; then the direct calculation using the VCVPF solution leads to the same decay rate as Lamb's exact solution at the long wave limit.

\subsection{Dissipation calculation (Lamb 1932, § 348)}

When gravity is important and $\boldsymbol{g}=-\boldsymbol{e}_{y} g$ where $y$ points upward, the energy equation (2.1) becomes

$$
\frac{\mathrm{d}}{\mathrm{d} t} \int_{V} \rho\left(\frac{1}{2} u^{2}+g y\right) \mathrm{d} V=\mathscr{P}-\mathscr{D}
$$

where $\mathscr{P}$ is the power of traction and $\mathscr{D}$ is the dissipation. In the present problem we look at functions periodic in $x$ with period $\lambda$ and $y=\eta(x, t)$ is the free surface and $-L \leqslant y \leqslant \eta, L \rightarrow \infty$. The gravity term gives rise to a potential energy

$$
\int_{V} \rho g y \mathrm{~d} V=\int_{0}^{\lambda} \frac{\rho g \eta^{2}}{2} \mathrm{~d} x
$$

Lamb notes that when the viscosity is neglected, the progressive wave may be represented by

$$
\phi=\alpha c \mathrm{e}^{k y} \cos k(x-c t), \quad \eta=\alpha \sin k(x-c t)
$$

where $c=\sqrt{g / k}$ for inviscid potential flow is the wave velocity. In fact, this relation between $\phi$ and $\eta$ holds only when $\alpha$ is independent of time. He notes that (6.3) will hold and the motion will persist, even with viscosity, provided that the surface stresses calculated on the potential flow are applied. In this case the dissipation in one period is

$$
\mathscr{D}=\mathscr{P}=2 \mu k^{3} \alpha^{2} c^{2} \lambda .
$$

In the free wave, with $\mathscr{P}=0$,

$$
\frac{\mathrm{d}}{\mathrm{d} t}\left(\int_{V} \rho \frac{u^{2}}{2} \mathrm{~d} V+\int_{0}^{\lambda} \frac{\rho g \eta^{2}}{2} \mathrm{~d} x\right)=\frac{\mathrm{d}}{\mathrm{d} t}\left(\frac{1}{2} \rho k \alpha^{2} c^{2} \lambda\right)=-\mathscr{D}=2 \mu k^{3} \alpha^{2} c^{2} \lambda .
$$

Equation (6.5) implies that

$$
\frac{\mathrm{d} \alpha}{\mathrm{d} t}=-2 \nu k^{2} \alpha
$$

Thus $-2 v k^{2}$ is the decay rate from the dissipation calculation. 
6.2. Direct calculation using VPF (Funada \& Joseph 2001)

The decay of free gravity waves can be treated as a stability problem using the theory of VPF. The stability analysis is a special case of the study of Kevin-Helmholtz stability given by Funada \& Joseph (2001). The governing equations are

$$
\begin{aligned}
& y \leqslant 0: \quad \boldsymbol{u}=\nabla \phi, \quad \nabla^{2} \phi=0, \\
& y=0: \begin{cases}\rho \frac{\partial \phi}{\partial t}=-p_{\mathrm{i}}-\rho g \eta & \text { Bernoulli equation, } \\
-p_{\mathrm{i}}+2 \mu \frac{\partial^{2} \phi}{\partial y^{2}}=0 & \text { normal stress balance, } \\
\frac{\partial \phi}{\partial y}=\frac{\partial \eta}{\partial t} & \text { kinematic condition. }\end{cases}
\end{aligned}
$$

After eliminating $p_{\mathrm{i}}$ and $\eta$ from (6.8) and applying the potential

$$
\phi=A \mathrm{e}^{k y+n t+\mathrm{i} k x},
$$

we find

$$
n=-v k^{2} \pm \mathrm{i} k \sqrt{g / k-v^{2} k^{2}} .
$$

Hence the amplitude of the wave decays at a rate

$$
\frac{\mathrm{d} \alpha}{\mathrm{d} t}=-v k^{2} \alpha
$$

one half of the rate given by (6.6). The wave speed is given by

$$
c=\sqrt{g / k-v^{2} k^{2}}
$$

which is slower than the inviscid wave speed $\sqrt{g / k}$ for $\nu k^{2}<\sqrt{g k}$. For very large values of $k, v k^{2} \gg \sqrt{g k}$ and short standing waves do not propagate but simply decay at a rate given by

$$
\frac{\mathrm{d} \alpha}{\mathrm{d} t}=-\frac{g}{2 v k} \alpha .
$$

\subsection{Exact solution (Lamb 1932, §349)}

Lamb gave an exact solution of the problem of decaying free gravity waves. It differs from the solutions using viscous potential flow just given, in that the conditions

$$
T_{x y}=0, \quad T_{y y}=\gamma \frac{\partial^{2} \eta}{\partial x^{2}}
$$

at the free surface (at $y=0$ ) are strictly enforced. (The surface tension $\gamma$ is not relevant in our discussion here and could be put to zero.)

The condition (6.14) cannot be satisfied by an irrotational flow. To accommodate vorticity, Lamb introduces a stream function $\psi$ and the solution is given by

$$
u=\frac{\partial \phi}{\partial x}+\frac{\partial \psi}{\partial y}, \quad v=\frac{\partial \phi}{\partial y}-\frac{\partial \psi}{\partial x}, \quad \frac{p}{\rho}=-\frac{\partial \phi}{\partial t}-g y,
$$

provided

$$
\nabla^{2} \phi=0, \quad \partial \psi / \partial t=\nu \nabla^{2} \psi
$$

No pressure term enters into the stream function equation; the pressure $p$ depends on the viscosity through the velocity potential. Lamb shows that the governing equations can be solved with normal nodes

$$
\phi=-A \mathrm{e}^{k y} \mathrm{e}^{\mathrm{i} k x+n t}, \quad \psi=-C \mathrm{e}^{m y} \mathrm{e}^{\mathrm{i} k x+n t}, \quad m^{2}=k^{2}+n / v
$$


provided

$$
\left(n+2 v k^{2}\right)^{2}+g k+\gamma^{\prime} k^{3}=4 v^{2} k^{3} m
$$

where $\gamma^{\prime}=\gamma / \rho$. When $v k^{2} \ll \sqrt{g k+\gamma^{\prime} k^{3}}$ (long waves) Lamb finds that

$$
n=-2 v k^{2} \pm \mathrm{i} \sqrt{g k+\gamma^{\prime} k^{3}} \text {. }
$$

The decay rate $-2 v k^{2}$ agrees with the dissipation approximation result (6.6). When $\nu k^{2} \gg \sqrt{g k+\gamma^{\prime} k^{3}}$ (short waves) and with $\gamma^{\prime}$ ignored,

$$
n=-\frac{g}{2 v k}
$$

which agrees with the decay rate (6.13) from the direct stability analysis using VPF. This limit is for very viscous fluid and negligible vorticity. Lamb says that this limit "... represents a slow creeping of the fluid towards a state of equilibrium with a horizontal surface."

The decay rate $-v k^{2}$ given by (6.11) is one-half of the exact solution at the long wave limit. This discrepancy is caused by the boundary layer at the free surface, which is not accounted for in the direct stability analysis using VPF. The vorticity $\omega$ is given by

$$
\omega=\frac{n}{v} C \mathrm{e}^{m y+\mathrm{i} k x+n t} .
$$

At the long wave limit, the vorticity is important in a thin boundary layer. Lamb gives an estimate of the thickness of the boundary layer, $2 \pi / \chi$, where $\chi=$ $\left(\sqrt{g k+\gamma^{\prime} k^{3}} / 2 v\right)^{1 / 2}$. The situation is different at the short wave limit, where the magnitude of the vorticity is very small and there is no sensible boundary layer. This explains why the decay rate arising from the direct calculation using VPF agrees with the exact solution at the short wave limit.

\subsection{Direct calculation using VCVPF}

At the long wave limit, a pressure correction to the irrotational pressure due to the vorticity layer is needed. We can solve for the viscous pressure correction from the linearized governing equation and prove that it is a harmonic series in this case. First we divide the velocity and pressure in the boundary layer near the interface into two parts

$$
\boldsymbol{u}=\boldsymbol{u}_{\mathrm{p}}+\boldsymbol{u}_{\mathrm{v}}, \quad p=p_{\mathrm{p}}+p_{\mathrm{v}},
$$

where the subscript $\mathrm{p}$ denotes potential solutions and $\mathrm{v}$ denotes viscous corrections. The linearized governing equation for $\left(\boldsymbol{u}_{\mathrm{v}}, p_{\mathrm{v}}\right)$ is

$$
\frac{\partial \boldsymbol{u}_{\mathrm{v}}}{\partial t}=-\frac{1}{\rho} \nabla p_{\mathrm{v}}+\nu \nabla^{2} \boldsymbol{u}_{\mathrm{v}} .
$$

We take the divergence of (6.23) and obtain $\nabla^{2} p_{\mathrm{v}}=0$. The solution of $p_{\mathrm{v}}$ can be expressed as a Fourier series

$$
-p_{\mathrm{v}}=\sum_{j=-\infty}^{\infty} C_{j} \mathrm{e}^{n t+j y+\mathrm{i} j x} .
$$

The zero shear stress condition at the free surface implies that $\partial u_{v x} / \partial y \sim O(1)$ and it follows from the continuity equation that $\partial u_{v y} / \partial y \sim O(\delta)$ where $\delta$ is the boundary layer thickness. The normal stress balance at $y=0$ is

$$
-p+2 \mu \frac{\partial^{2} \phi}{\partial y^{2}}=0,
$$


where the surface tension and the term $2 \mu \partial u_{\mathrm{v} y} / \partial y$ are ignored. Equation (6.25) can be written as

$$
\rho \frac{\partial \phi}{\partial t}+\rho g \eta-p_{\mathrm{v}}+2 \mu \frac{\partial^{2} \phi}{\partial y^{2}}=0 .
$$

Substituting the expressions for $\phi$ (6.9) and $p_{\mathrm{v}}$ (6.24) into (6.26), we have

$$
\left(\rho n A+\rho \frac{g k}{n} A+2 \mu k^{2} A+C_{k}\right) \mathrm{e}^{n t+\mathrm{i} k x}+\sum_{j \neq k} C_{j} \mathrm{e}^{n t+\mathrm{i} j x}=0 .
$$

By orthogonality, we obtain

$$
\rho n A+\rho \frac{g k}{n} A+2 \mu k^{2} A+C_{k}=0 \quad \text { and } \quad C_{j}=0 \quad \text { if } \quad j \neq k .
$$

The constant $C_{k}$ can be determined using (2.18). We list the velocities and stresses at $y=0$ evaluated on the potential:

$$
\left.\begin{array}{l}
u_{\mathrm{p} x}=\mathrm{i} k A \mathrm{e}^{n t+\mathrm{i} k x}, \quad u_{\mathrm{p} y}=k A \mathrm{e}^{n t+\mathrm{i} k x} \\
\tau_{y y}=2 \mu k^{2} A \mathrm{e}^{n t+\mathrm{i} k x}, \quad \tau_{x y}=\mathrm{i} 2 \mu k^{2} A \mathrm{e}^{n t+\mathrm{i} k x} .
\end{array}\right\}
$$

The power of the pressure correction and power of the shear stress are

$$
\int_{0}^{\lambda} u_{\mathrm{p} y}^{*}\left(-p_{\mathrm{v}}\right) \mathrm{d} x=C_{k} A k \lambda, \quad \mathscr{P}_{\mathrm{s}}=\int_{0}^{\lambda} u_{\mathrm{p} x}^{*}\left(\tau_{x y}\right) \mathrm{d} x=2 \mu A^{2} k^{3} \lambda,
$$

where the asterisk denotes conjugate variables. It follows that

$$
C_{k}=2 \mu k^{2} A \text { and }-p_{\mathrm{v}}=2 \mu k^{2} A \mathrm{e}^{n t+k y+\mathrm{i} k x} .
$$

Inserting the value of $C_{k}$ into (6.28), we have

$$
\rho n+\rho \frac{g k}{n}+2 \mu k^{2}+2 \mu k^{2}=0
$$

and the solution for the potential is

$$
\left.\phi=A \mathrm{e}^{k y} \mathrm{e}^{-2 v k^{2} t} \mathrm{e}^{\mathrm{i} k(x \pm t} \sqrt{g / k-4 v^{2} k^{2}}\right) .
$$

The amplitude of the wave decays at a rate $-2 v k^{2}$ which agrees with the dissipation result and Lamb's exact solution at the long wave limit.

This analysis of the pressure correction of progressive waves can be easily modified to obtain the pressure correction of standing waves. If we use the potential $\phi=\left((\mathrm{d} a / \mathrm{d} t) \mathrm{e}^{k y} / k\right) \cos k x$ for standing waves, we can obtain

$$
-p_{\mathrm{v}}=2 \mu k(\mathrm{~d} a / \mathrm{d} t) \mathrm{e}^{k y} \cos k x,
$$

which is exactly the same as the pressure correction obtained by Prosperetti (1976) using a different method.

\section{Conclusion}

This paper concerns viscous potential flow for problems with a free surface. When treating potential flow it is neither necessary nor useful to put the viscosity to zero. The theory of viscous potential flow can give an accurate description of the fluid dynamics when the vorticity is essentially zero or is confined to a narrow layer near 
the boundary. There are many situations in which the viscosity is very large and the vorticity is small and not important. Some problems of this type have been analysed by the dissipation method which has been used to describe motions of bubbles and drops and the effects of viscosity on the decay of irrotational waves. The dissipation method involves the computation of viscous stresses using the irrotational strain rate; vorticity and vorticity layers do not enter these computations of drag or decay. Another way to approach these problems is by direct application of viscous potential flow. This method works well only when vorticity layers are unimportant. When there are vorticity layers, the direct method can be corrected by adding a pressure correction $p_{\mathrm{v}}$ to the irrotational pressure $p_{\mathrm{i}}$; the viscous normal stress is computed for the irrotational motion and only the pressure is corrected for vorticity. The pressure correction is presumed to arise from a boundary layer of vorticity induced by the unbalanced irrotational shear stress. Boundary layer analyses have appeared in the literature, but have not achieved a pressure correction formula which would lead to the same drag on a gas bubble rising in a viscous liquid at high Reynolds number as the drag calculated by the dissipation method.

Here, we have derived an explicit relation between the pressure correction and the irrotational shear stress at the interface. A periodic or finite pressure correction may be expanded as a harmonic series. We may explicitly compute the coefficient of the principal mode in the series which is matched to the velocity potential. The other modes do not enter into the expression for the drag on bubbles and drops. They vanish in the case of free gravity waves. Our pressure correction formula is applied to cases for which dissipation results are computed in the literature; when the irrotational pressure is corrected, our direct computation gives the same result as the dissipation computation. Our pressure corrections are confirmed by independent results in the case of a rising spherical gas bubble (Kang \& Leal 1988) and in the case of the decay of standing free waves (Prosperetti 1976). We also computed the pressure corrections for a rising oblate ellipsoidal gas bubble and a rising spherical liquid drop, for which no independent results are available for comparison. They reduce to the pressure correction for a rising spherical gas bubble at the limit, which provides indirect corroboration for these pressure corrections.

The pressure correction formula shows that these corrections are not important in problems in which the irrotational shear stress is small or zero. In these cases, listed below, the direct application of viscous potential flow leads to outstanding results. There are other cases, discussed in this paper, in which the pressure correction is needed.

(i) The Rayleigh-Plesset bubble. This is an exact viscous potential flow solution of the Navier-Stokes equations. This bubble describes purely radial motions in which the irrotational shear stress is identically zero.

(ii) The rise velocity of a spherical cap bubble. Large gas bubbles do not stay spherical; instead they take the lenticular shape of a spherical cap bubble. The vorticity layers do not appear to strongly affect spherical cap bubbles. Davies \& Taylor (1950) showed that the rise velocity of such a bubble could be obtained from a local analysis without using a drag balance, noting that the nose of the bubble is spherical as a result of the pressure generated by motion, without surface tension. Joseph (2003) generalized their inviscid potential flow result to include effects of viscosity, surface tension and the deviation of the bubble nose from sphericity using viscous potential flow and he obtained a hyperbolic drag law

$$
C_{D}=6+32 / R .
$$


The Davies-Taylor result $C_{D}=6$, and the formula (7.1) of viscous potential flow, are in excellent agreement with the experimental results of Bhaga \& Webber (1981) for small and large $R$. The $32 / R$ term is correct because the irrotational shear stress vanishes at the stagnation point and is small near the stagnation point at the nose of the rising bubble.

(iii) Stability limits and maximum growth rates for Rayleigh-Taylor instability of viscous fluids (Joseph, Belanger \& Beavers 1999) and viscoelastic fluids (Joseph, Beavers $\&$ Funada 2002). These results depend very strongly on the viscosity and viscoelastic parameters. For this problem there is at most a few percent difference between the results of viscous and viscoelastic potential flow and the exact solution. The reason for the excellent results of the potential flow analysis is that the irrotational shear stress of the basic flow vanishes identically.

The exact solution of Lamb $(1932, \S 349)$ tends to the irrotational solution at the short wave limit in the gravity waves problem; the zero traction condition at the surface is satisfied by a non-propagating wave which tends monotonically to $y=0$. This is another case in which the irrotational solution is exact.

Viscous potential flow will not work when vorticity is important; when it is not important, potential flow can be used but the viscosity should not be put to zero.

This work was supported in part by the NSF under grants from Chemical Transport Systems and the DOE (engineering research program of the Dept. of Basic Engineering Sciences). We wish to thank Professor G. I. Barenblatt for his interest and helpful discussions of topics treated in this paper.

\section{REFERENCES}

Batchelor, G. K. 1967 Introduction to Fluid Dynamics. Cambridge University Press.

Bhaga, T. \& Webber, M. 1981 Bubbles in viscous liquids: shapes, wakes and velocities. J. Fluid Mech. 105, 61-85.

Davies, R. M. \& TAYLoR, G. I. 1950 The mechanics of large bubbles rising through liquids in tubes. Proc. R. Soc. Lond. A 200, 375-390.

FunAda, T. \& JosePh, D. D. 2001 Viscous potential flow analysis of Kelvin-Helmholtz instability in a channel. J. Fluid Mech. 445, 263-283.

Harper, J. F. \& Moore, D. W. 1968 The motion of a spherical liquid drop at high Reynolds number. J. Fluid Mech. 32, 367-391.

Joseph, D. D. 2003 Rise velocity of a spherical cap bubble. J. Fluid Mech. 488, 213-223.

Joseph, D. D., Beavers, G. S. \& FunAdA, T. 2002 Rayleigh-Taylor instability of viscoelastic drops at high Weber numbers. J. Fluid Mech. 453, 109-132.

Joseph, D. D., Belanger, J. \& Beavers, G. S. 1999 Breakup of a liquid drop suddenly exposed to a high-speed airstream. Intl J. Multiphase Flow 25, 1263-1303.

Joseph, D. D. \& LiaO, T. Y. 1994 Potential flows of viscous and viscoelastic fluids. J. Fluid Mech. 265, 1-23.

Kang, I. S. \& Leal, L. G. 1988 The drag coefficient for a spherical bubble in a uniform streaming flow. Phys. Fluids 31, 233-237.

LAmB, H. 1932 Hydrodynamics, 6th edn. Cambridge University Press. (Reprinted by Dover, 1945)

Levich, V. G. 1949 The motion of bubbles at high Reynolds numbers. Zh. Ekspe. Teor. Fiz. 19, 18.

Moore, D. W. 1959 The rise of a gas bubble in a viscous liquid. J. Fluid Mech. 6, 113-130.

Moore, D. W. 1963 The boundary layer on a spherical gas bubble. J. Fluid Mech. 16, 161-176.

Moore, D. W. 1965 The velocity of rise of distorted gas bubbles in a liquid of small viscosity. J. Fluid Mech. 23, 749-766.

Prosperetri, A. 1976 Viscous effects on small-amplitude surface waves. Phys. Fluids 19, 195-203.

ZIEREP, J. 1984 Viscous potential flow. Arch. Mech. 36, 127-133, Warszawa. 